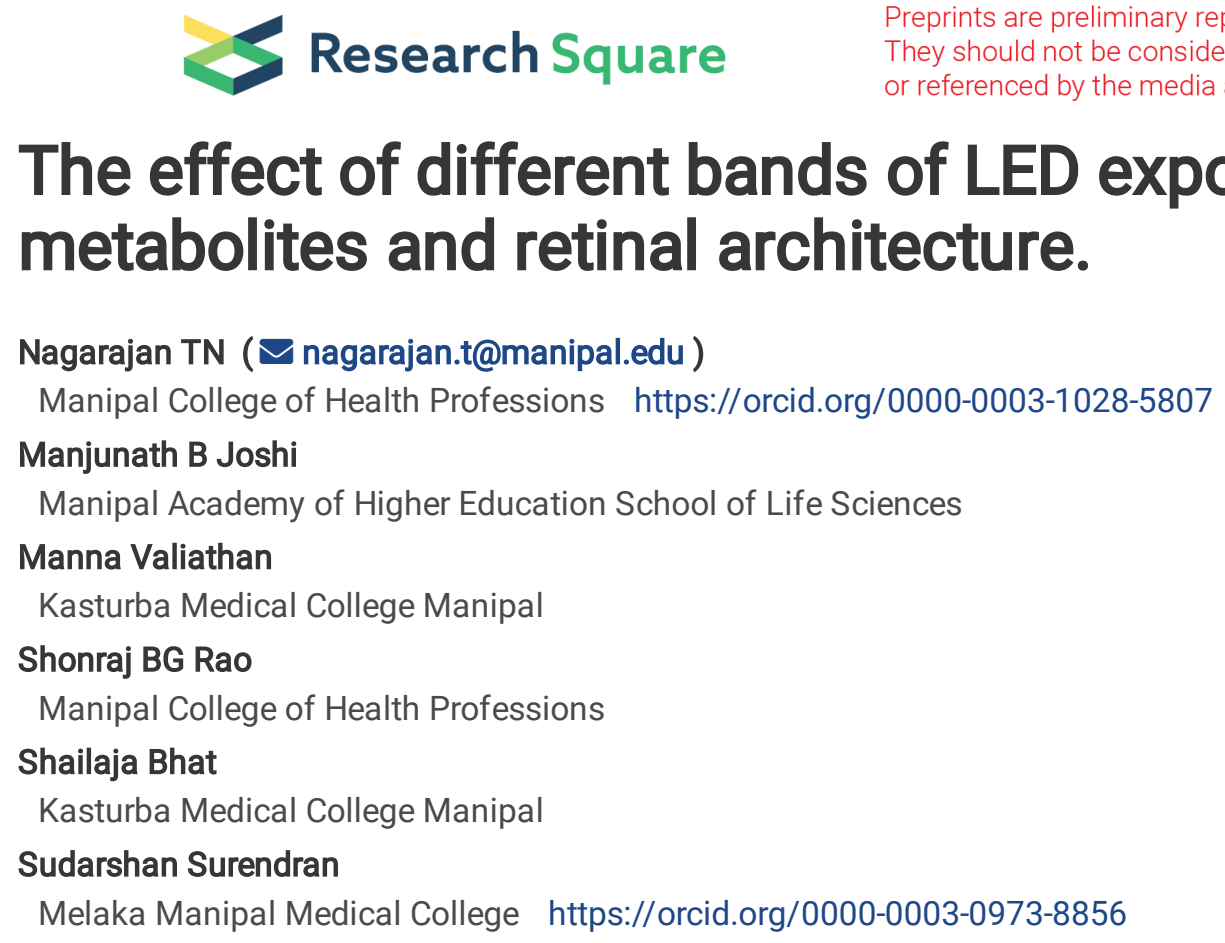

\title{
The effect of different bands of LED exposure on vitreous metabolites and retinal architecture.
}

\section{Research Article}

Keywords: Vitreous metabolites, Apoptosis, Immunofluorescence, CASPASE-3, Retinae, Light-induced damage, Amino acids Posted Date: February 18th, 2022

DOI: https://doi.org/10.21203/rs.3.rs-1325637/v1

License: @ (i) This work is licensed under a Creative Commons Attribution 4.0 International License. Read Full License 


\section{Abstract}

The accumulating experimental evidence indicates that exposure to blue and white LED light leads to damage in the visual system against short-term exposure. Chronic exposure, adaptive responses to light and self-protective mechanisms against LED light exposures need to be explored and would be essential to know the repercussions of LED radiations on vitreous metabolites. A total of 24 male Wistar rats were used in our study, which was divided into four groups ( $n=6$ in each group). Three groups were exposed to either blue, white, or yellow LED light for 90 days (12:12 light-dark cycle routine) with uniform illumination (450-500 lux). Control rats were maintained under standard laboratory conditions. Post-exposure the vitreous was removed for mass spectrometry and retinal tissues for immunofluorescence and H\&E staining. The thickness of the retina decreased in blue and white light exposure animals compared with controls, whereas the yellow light exposure group showed an increase in thickness $(p<0.001)$. The number of apoptotic cells was significantly lower in controls compared to light-exposed groups $(p<0.001)$ and $(p<0.001)$. Altered metabolites were observed in light exposure groups particularly in $D$-alanine, taurine, $D$-serin $(p<0.05$ and lysine $(p<0.001)$. The self-protective or reworking system in the chronic light exposure could be dazed and drop the ability to compensate for the defending mechanism. This might fail to maintain the metabolomic structural integrity of the vitreous and retina.

\section{Introduction}

Light plays a crucial role in visual perception from a non-ionizing visible light spectrum between 380-700nm [1]. Environmental light plays a significant role in zeitgebers [2,3]. The retinal ganglion cell layer and photoreceptors provide vital information about ambient illumination and light-dark cycles to visual and non-visual higher cortical centres [4]. Non-natural light is an essential source in contemporary life, a large segment of the population is exposed to a different type of light-emitting diodes (LEDs), and it is cast off globally for interior and exterior lighting and rudimentary lighting components of digital devices.

The blue range of the visible spectrum has high energy that can penetrate tissues, which is associated with the occurrence of malignant melanoma compared to other wavelengths of the visible spectrum in animal models [5]. LED radiation diminishes the capability of cellular mechanism and increases apoptosis and it damages DNA by excessive production of ROS [6, 7]. These mechanisms are activated by chromophores within the retinal cells, they are intrinsically sensitive retinal ganglion cells [8]. These LEDs can alter ocular (retinal) and other biological tissues $[4,6,9,10]$. Excessive exposure to light can alter physiological rhythms like sleepiness, alertness and increase cortisol levels [11, 12].

Radiation-induced retinal damage can occur either through photo-vaporization or photochemical mechanisms $[13,14]$. Photovaporization results in disruption of tissue by short-wavelength light exposure with high irradiance level [15]. The photochemical injury occurs when tissue temperature increases due to the entrapment of thermal energy into a tissue molecule which could result in retinal damage [16]. Biological chromophores of retinal pigmented epithelial cells can captivate the photons of light from visible spectrum 380-780nm. These absorptions are necessary for visual functions, but peak energetic radiations (violet to blue 400$500 \mathrm{~nm}$ ) can lead to a lethal consequence to retinal cells $[17,18]$. These absorbed excited states of electrons can return to the inhibited state by dissipating the superfluous energy, and the energy molecules can break a bond in another molecule through a direct exchange of electron or hydrogen-producing reactive oxygen species (ROS)[19, 20]. The interaction of these chromophores with photons of light might generate ROS which might damage the amino acids, lipids, and DNA [21].

The visual system is exposed to a limited time to LED lights, of different spectra as its technology are blooming now to decrease the energy consumption and $\mathrm{CO}_{2}$ emission. These technologies eventually replaced standard lighting systems of indoor and outdoor. The white and blue LEDs have high energetic characteristics compared with other traditional light sources, so the potential ocular and general risks of these LEDs are widely explored [4, 19,22-26]. Inference from these studies cannot be generalized because few studies suggested that low levels of blue light for a longer duration (few months to years) are needed to determine the effects of blue light on the visual system [27]. Considering this we have hypothesized that chronic exposure might induce a compensatory mechanism, stimulated by an increased insult to ocular tissues and fluid. Hence, we aimed at understanding the relationship between chronic light exposure and alterations in the vitreous metabolites and retina structures in Wistar rats.

\section{Methodology}


The Manipal Academy of Higher Education, Kasturba Medical College animal ethics committee approved the experimental protocol (IAEC/02/2017). Animal handling and investigational procedures were carried out as per IAEC and CPCSEA (No:94/PO/Re Bi/5/99/ CPCSEA) guidelines. The healthy animals $(n=24)$ are divided into three experimental groups ( $n=6$ in each group), one serving as an (unexposed) control group $(n=6)$. We have included healthy rats for the study and the control rat's retinas were selected from the same pool of rats. However, it's safe to assume that the experimental control rats will also not have any degeneration. Both eyes of rats were exposed to blue, white, and yellow LED light with the maintenance of homogeneousness and intensity of light. The light standard was measured and standardized using Asensetek Lighting Passport Pro, New Taipei City, Taiwan[28]. The spectral sensitivity of different wavelengths of light visible spectrum 400-780nm) used for this experiment (450-500 lux) light. The spectral properties of light transmittance of the front surface of light, scotopic sensitivity, flicker index and melatonin suppression of each light were measured. The total light exposure per day of (450-500 lux). The height from the source to the animals (cage) was 50 $\mathrm{cm}$ and the exposure time (12:12 dark \& light cycle routine for 90 days) was matched with the nocturnal time of the rodents. The light exposure group animals were subjected to the blue LED $(400-490 \mathrm{~nm})$, white LED (380-780nm) and yellow LED (400-780nm) light exposure (Ack LED Panels, 3W, Epistar, ES-EMBCF22L-A, InGan -series Blue LED chip, ES-SMYLPX42C, UHB-PX, AIGalnP PNseries LED Chip). The light source was fitted on the top of the cage $(L=100 \mathrm{~cm}, W=70 \mathrm{~cm}$ and $H=50 \mathrm{~cm})$ the mounting of the light kept it at $50 \mathrm{cms}$ ( $100 \%$ system level light output at standard operating voltage range, the total light output of the cage at the testing area (510 lumens). Post-exposure, all animals were sacrificed with a lethal dose of pentobarbital intraperitoneal injection (i.p. $100 \mathrm{mg} / \mathrm{kg}$ ) (Euthasol®) and xylazine $\left(10 \mathrm{mg} / \mathrm{kg}\right.$ ) (Proxylaz $\left.{ }^{\circledR}\right)$. Then eyes were enucleated by using watchmaker forceps (number 5) and Sklar's blunt enucleation scissors. Immediately after enucleation, the vitreous was extracted by aspiration (21gauge hollow needle) technique and stored in $-20^{\circ} \mathrm{C}$ for LC-MS and the retinal tissue stored in $4 \%$ paraformaldehyde ( 7 days) for immunofluorescence (IF) and Hematoxylin and eosin (H\&E) stain.

\section{Rat whole vitreous:}

For the analysis of the chronic light exposure model, the whole vitreous sample was collected by aspiration technique. The samples were sonicated and stored at $-20^{\circ} \mathrm{C}$ until LC-MS analysis.

\section{Vitreous Metabolomic Analysis}

Untargeted quantitative metabolomics was used to analyze the vitreous fluid samples using an LC-MS Agilent LC-QTOF system (Agilent Technologies, Santa Clara, CA, USA). It consisted of an Agilent 1200 LC system coupled online with an Agilent 6520 timeof-flight mass spectrometer. The vitreous samples were defrosted on ice, vortexed, and centrifuged at 12,000 rpm for $15 \mathrm{~min}$. An 8 $\mu \mathrm{l}$ aliquot was injected into an Agilent $1290 \mathrm{LC}$ system coupled to an ESI-Q-TOF instrument (Agilent 6520, Agilent Technologies, Santa Clara, CA, USA). HPLC column (Phenomenex, Torrance, CA, USA (P/No:00G-4601-E0; Desc: Kinetex 5 $\mu m$ C18 100A; Size: LC Column 250X $4.6 \mathrm{~mm}$; S/No H18-343854; B/No 5701-0060) was maintained was at $25^{\circ} \mathrm{C}$. The injected vitreous data were collected in the positive mode of electrospray ionization (ESI) technique. Basic and neutral metabolites were eluted in positive mode using subsequent gradient at $400 \mu \mathrm{l} / \mathrm{min}$ using mobile phase A: $0.1 \%$ formic acid in water and mobile phase B: $0.1 \%$ formic acid in $90 \%$ acetonitrile ( $2 \%$ to $98 \%$ B in 25 minutes, $98 \%$ B for 10 minutes and equilibrated to $2 \%$ B for $10 \mathrm{~min}$ ). ESI spray voltage was maintained at $3.5 \mathrm{kV}$ with $\mathrm{MS}$ interface capillary at $350^{\circ} \mathrm{C}$. Fragmentor was set at $140 \mathrm{~V}$. Drying gas and nebulizer were maintained at 8 litres/minute and pressure of 40 psig respectively. Data was acquired at an acquisition rate of $2 \mathrm{~Hz}$ in a range of 50-1700 m/z. Based on precise mass value and retention time, an untargeted mass spectrometer approach was employed for confirmation of the most abundant and significant metabolites.

\section{Data processing and analysis:}

The raw data obtained from each run was processed using a molecular feature extraction tool in Qualitative Mass Hunter Analysis Software B.04.00 (Agilent Technologies). The data files containing monoisotopic mass, respective abundance and retention time were used for data alignment and filtering in Mass Profiler Plus software (MPP) (Agilent Technologies, version B.12.5). Raw data files were segregated aligned, transformed to $\log 10$, and baselined to the median of all samples in MPP. Features that were present in at least $75 \%$ of individuals in each group were considered for further analysis. Partial least square discriminant analysis (PLSDA) was performed on metabolome data to discriminate the study groups.Compounds were identified in METLIN and HMDB databases based on isotopic pattern distribution and accurate mass within a specified tolerance (15 ppm error). Peaks obtained 
from raw MS data were aligned and subjected to bioinformatics and statistical analysis. The metabolite peaks were attained in NC, BLE, WLE and YLE groups separately (Figure 1:).

\section{Retinal Immunostaining:}

The twenty-four retinal tissues were embedded in $4 \%$ paraformaldehyde immediately after the following enucleation. The retinal tissues were sectioned after calibrating the cryostat for $40 \mu$ thickness and the sections were mounted on the gelatin-coated slides serially. Frozen sections (Leica cm3050 s) of 40 microns thickness were made in the sagittal plane and kept at $-80^{\circ} \mathrm{C}$ before further processing. Sections were fixed with $2 \%$ paraformaldehyde in $0.01 \mathrm{M}$ phosphate buffer solution at $24^{\circ} \mathrm{C}$ for 1 hour, followed by incubation with $1 \% \mathrm{H}_{2} \mathrm{O}_{2}, 2 \%$ sodium azide, $0.1 \%$ saponin, $10 \mathrm{mM}$ (4-(2-hydroxyethyl)-1-piperazineethanesulfonic acid (HEPES) in EBSS -saponin for 36 hours at $24^{\circ} \mathrm{C}$ in the dark. Sections were then rinsed and incubated in a moist chamber overnight at $24^{\circ} \mathrm{C}$ with primary rabbit polyclonal to active + pro Caspase 3 antibody directed against cleaved caspase-3 (1:200 aliquoted $4 \mu$ l of antibody is added to $1.6 \mathrm{ml}$ of PBS.TX) with working buffer and approximately $200 \mu \mathrm{l}$ of diluted (Abcam, Cambridge, UK). The retinal tissue sections were washed and incubated with a secondary antibody (1:100, Anti-Rabbit lgG (whole molecule) F(ab)2 fragment-Cy3 antibody, Abcam) for 16 hours at $2^{\circ} \mathrm{C}$.

Immunofluorescence imaging and quantification: Caspase-3 (active + pro Caspase 3) stained retinae were imaged using a Dmi8SP8 Confocal Microscope (Leica Microsystems) using a $63 \mathrm{X}$ objective. The laser power offset and gain, and other acquisition parameters were determined by using isotopic control samples. The values of these parameters were then fixed across all retinal samples. Retinal mosaics were created in Adobe Photoshop 7.0 (Mountain View, CA, USA). The area of each retinal layer was segmented by a masked observer, and the average intensity was calculated after subtracting the background using ImageJ software (To overcome the background intensity staining we have accounted brightness in each layer, and it was expressed relative intensity to the entire retina, and the total brightness was averaged). A total of 12 images, 2 from each eye were analyzed in each group and the intensity of IF was quantified in a masked method.

\section{Measurement of hematoxylin and eosin-}

Measurement of hematoxylin and eosin-stained retinal layers was conducted as follows. Images of a sagittal plane containing the optic nerve on paraffin-embedded blocks of H\&E images were taken using a Motic Images Plus 3.0 for Windows 32 (2021-10-29) Microscope (Leica Microsystems, Germany) at 40x magnification then Retinal mosaics were created in Adobe Photoshop 7.0 (Mountain View, CA, USA). Images were analyzed with the built-in measuring tool in Image-J software. The thicknesses of the whole retina (excluding the outer segments) and the ganglion cell layer (GCL), inner plexiform layer (IPL), inner nuclear layer (INL), outer plexiform layer (OPL) and outer nuclear layer (ONL) were measured for each layer in each picture in three different areas. Data were collected in Excel and then imported to R software (version 3.6.3) for statistical analysis

\section{Data preparation and statistical application}

The LC-MS/MS spectral region 5.00 ppm was segmented into bins of $0.05 \mathrm{ppm}$ width Agilent pro software. The region anything above 5.00 ppm error was excluded from the analysis as it might have a remnant noise signal. A total column (bins) of metabolites were obtained and the integrated area within each bin was normalized to a constant sum of 500 for each range to minimize the effects of variable concentration among different samples.

\section{Results}

Different band of LED persuaded retinal insult: As we showed in figure 2: after 90 days oflight exposure the retinal layers showed depletion with marked proliferation, increased cellularity and enlarged cells with vacuolation of ganglion cell layer (GCL), and it became thinnerand disorganized, and more hyperchromatic nucleicells were observed in BLE and WLE groups. The GCL of the retinas inYLE was slightly disarrayed with nuclear enlargement and outer nuclear layer (ONL) thickness was increased with a focal enlargement (Figure 2:). The ONL thicknesswas decreased in BLE and WLE groups (Table 1:). We found there were varying notches of decreased thickness in the outer nuclear layer (ONL) in the BLE and WLE groups in contrast with YLE and NC. There was no significant difference was found in the INL thickness in all groups ( $p<0.01)$. As shown in (Figure 2:) the nucleolar damage, 
including pyknosis, was distinct with detached IS and OS structures and irregular arrangements of the photoreceptors in groups BLE and WLE when compared with the YLE and NC groups.

The stained retinal sections were obtained in the dorsal-ventral axis for immunofluorescence (Figure 3A:) (IF) quantification. The whole retinal tissue section was selected at different peculiarities, for cell count and the total number of stained nuclei was counted in a 200- $\mu \mathrm{m}$-long tissue section. For every retinal location, the amount of apoptosis in the NC group of retinae was significantly lower than in the light-exposed groups (ANOVA) (Figure 3B:). Besides, statistically significant differences were found in light exposure groups of rats $(p<0.01)$ (Figure 3B:).

The retina showed a significant change after 90 days across the light exposure groups (Figure 3A:). IF analysis of CASPASE-3 expression showed a reorganization of morphological structures in $\mathrm{BL}$ and WL in comparison with NC whereas no obvious morphological changes were noticed in inner retinal layers and significant damage occurred in ONL in the YLE group using Cy-3 staining. The percentage of apoptotic cells was increased BLE and YLE in GCL, INL, ONL $(p<0.001)$ and inner and outer segments of photoreceptor and IPL are relatively reduced in the YLE and NC group. However, the apoptosis was $86 \%, 84 \%$, and $66 \%$ for BLE, WLE and YLE -light exposure tissues respectively $(\mathrm{p}<0.001)$ (Figure 3B:). Four regions of interest in every retinal tissue section were selected at different peculiarities, for cell count and the total number of stained nuclei was counted in a $200-\mu m-l o n g$ tissue section. The amount of apoptosis in the NC group of retinae was significantly lower than in the light-exposed group's rats ( $p<0.001)$ (Figure 3B:). Besides, statistically significant differences were found in light exposure groups of rats $(p<0.001)$ and $(p<0.001)$ (Figure 3B:). However, no self-protective or recovery was observed based on the hypothesis. The acquired images from the posterior pole within $1 \mathrm{~mm}$ of the optic nerve and the phototoxicity also were observed in the region of interest (ROI).

\section{Metabolomic amendment of chronic light exposure and reprogramming.}

A total of 24 vitreous samples, withdrawn from light exposure (BLE, WLE and YLE) and control group (Time 10:00 AM-10:30 AM IST) were analyzed. We got 20884 hits across the groups and these molecules were filtered out by frequency out of these 459 molecules which were presented across the samples. These molecules were considered to perform further analysis (one-way ANOVA) to observe the significance.

243 molecules were statistically significant $(\mathrm{p}<0.05)$ for the entity of 2.0 -fold change $(\mathrm{FC})$. Further, we did differential expression analysis using a t-test against zero, kept the $p$-value as asymptotic across the samples with an FC of 3.1. Out of total (20884) molecules 1521 were statistically significant to specific 907 molecules $(p<0.05), 348$ molecules $(p<0.02), 169$ molecules $(p<0.001)$, 82 molecules $(p<0.0001)$ and 20 molecules were $(p<0.00001)$ exhibited definite statistical alteration.

\section{PCA and PLS-DA}

We steered Principal component analysis (PCA) on all detected metabolites. The parting of the NC, BLE, WLE and YLE groups demonstrated their difference in metabolomic expression. Most metabolites with extreme abundance were certainly isolated in light groups. Further, we did a summary of frequency normalization for abundance values (log10) for across the groups out of it $99 \%$ of samples were normally distributed and outliers were removed in each group. Only significant metabolites into account, the molecules in the same group clustered to each other which stated less difference among the light exposure group.

Through partial least square discriminant analysis, we found the pattern of metabolomics, with a sensitivity of $90 \%$ and a specificity of $87 \%$. Notably, the amino acids and small molecule levels were elevated in the BLE and WLE groups. A clear difference in metabolomic expression of the BLE and WLE, YLE groups and the control group is displayed where there is a significant cluster of metabolites were observed between the groups.

Further to comprehend we heat map (Figure 4:) representation for the significant (243 molecules $(p<0.05)$ ) metabolites and we observed a clear upregulation of molecules in BLE, WLE and YLE where these molecules were in normal range/downregulated in NC. Having three groups of light exposure (BLE, WLE and YLE) from age-matched controls (NC). The BLE\&WLE exposure groups have been vital to show the specificity of these biomarkers to vitreous. Some metabolites were perturbed in all groups. Untargeted metabolomics by LC-MS/MS revealed 20 metabolites that are biologically relevant to ocular fluid. Thus, we next sought to validate our findings using the targeted analysis to quantify the concentrations of these metabolites. As well as targeting the metabolites dysregulated in BLE, WLE and YLE samples, we expanded the targeted analysis to include metabolites from related metabolic 
pathways, to determine the biological relevance of our findings using METLIN, Agilent pro and HMDB databases. The focused analysis was carried out using authentic standards to obtain accurate fold changes of the metabolites in the extracted samples. It was confirmed that 20 metabolites were statistically significant.

To further understand the perturbed metabolomic alterations, develop over the light exposure period of 90 days in Wistar rats, we performed one-way ANOVA and these values were compared across the groups. The p-values $<0.05$ considered as significant and significantly altered metabolites between the groups are plotted in Table 2: using Tukey HSD post-hoc test.

Pathway enrichment (figure 5:) analysis was performed for the very significant 20 metabolites in the NC, BLE, WLE and YLE groups using the MetaboAnalyst 4.0 program. The analysis revealed that arginine, aspartate glutamate and taurine metabolites were the most perturbed pathways in light exposure conditions (Figure 5:). The analysis revealed that alanine, aspartate, and glutamate ( $p=$ 0.0600), Pathway analysis of the significant metabolites identified for the comparison among BLE revealed significant enrichment for aspartate $(p=0.0068)$, glutathione $(p=0.0263)$, glycine $(p=0.00436)$, glutamate $(p=0.054)$, leucine and taurine $(p=0.007)$ and taurine and hypotaurine degradation (Figure 5:) were the most perturbed pathways in BLE and WLE conditions. This augments a growing frame of evidence to suggest that photo-chemical metabolism is particularly compromised in LE animals that potentially disrupts the taurine and hypotaurine dysregulation pathway.

\section{Discussion}

This study reveals 90 days of (12:12 hours) cyclic LED light exposure to rat's eye resulted in vitreous metabolomic changes and retinal structure alterations. These observations might be related to oxidative stress within the retinal tissue consistent with the aforementioned findings of short exposure especially a few days to months $[6,13,29,30]$.

LC-MS showed obvious changes in the vitreous metabolites after 90 days of light exposure (BLE, WLE and YLE) and some of these changes, like upregulated amino acids (AA) and lipids were more intense in BLE and WLE groups while others, like the increase in levels of AA, were observed from YLE. Light exposed vitreous showed several notable differences in the spreading silhouettes of the perceived metabolites between LED light exposure groups and normal controls of the vitreous fluid, predominantly, amino acids and proteins. Inadvertently, the role of altered metabolites and excitotoxic potential to light-induced damage in the vitreous is not well documented. It has been involved in a few ischemic induced ocular disease conditions including retinal vessel occlusion, glaucoma, and diabetic retinopathy [31,32]. The glutamate levels were altered in BLE and WLE groups which could also be a triggering factor for retinal apoptosis $[33,34]$. Glutamate is primarily mediated by overstimulation of the NMDA subtype of glutamate receptor, triggering an increase in intracellular calcium and initiating a cascade of events that finally lead to cell death/apoptosis, depending on glutamate levels [35]. The eccentricities in glutamate metabolism result in elevation of the extracellular concentration of glutamate which might increase the risk of excitotoxicity [33]. This indicates that failure in upholding glutamate homeostasis might lead to ocular toxicity [33].

The ganglion cell layer (GCL) thickness was drastically reduced in BLE and WLE groups, which might be due to the elevation of vitreal glutamate levels [36]. We noticed that metabolites of the vitreous fluid were dysregulated in the light exposure (BLE and WLE) groups, which could be due to a shift of glycolysis' products, such as fructose 1,6 bisphosphate (F1,6BP; a product of glycolysis) and citrate, to the inner retina, while glucose 6 phosphate (G6P) was detected at high intensities both in the inner and outer retina [37]. The up-regulated metabolites in BLE and WLE groups could be due to metabolomic requirements to withstand or protect from light-induced damage. Studies have demonstrated the importance of cytokines induced protective mechanism of photoreceptors in light-induced damage against constant exposure [38,39] and stated that this could be due to survival-promoting genes and proteins that might alter during retinal light adaptation to a new bright light environment in the BLE and WLE groups. The identified molecules from Alvarez et al., 2001 which can protect against light-induced damage were up-regulated contemporaneously [40].

Interestingly, de la Barca et al., 2017 studied the effect of light stress in the retinal metabolomes and found that the mechanisms of light preconditioning (pre-exposure to moderate light before intense light) remained unknown, even though they seemed to have a self-protective effect. The light-induced changes in vitreous metabolites such as amino acids and lipids could likely involve nitric oxide-related signalling pathways [14,37]. Post-acute bright light exposure, the retinal proteins expressions were up-regulated in particular glial $®$ brillary acidic protein, heme oxygenase and heat-shock protein as well as cytokines [39, $41-43]$. Beneath a

Page 6/17 
constant light exposure condition, the endurance of the alteration of vitreous metabolites and its concentration revealed that this could be an endogenous oscillator, it correlates with previous experiments on aqueous proteins[44]. The upregulated metabolites concentration noticed in light exposure groups (BLE and WLE) (Fig. 4:) could be related to light responses in ocular bounded tissues or linked cellular restoration[44] and antioxidant protection to retinal tissues[45]. Abundant proteins remained to play a major role in modifying such light damage[46]. Our current experiment suggests that the degree of susceptibility to light damage in tissues adjoining to the vitreous may also be contingent upon time and light dependence.

The increase in period of cumulative and constant light exposure for 90 days on vitreous fluid, the self-protective or reworking system in light (12:12 light-dark cycle) period could be dazed and might drop the ability to compensate for the defending mechanism, and thus might fail to maintain the metabolomic structural integrity. Such mechanisms increase post LED exposure if the compensatory mechanism is failed due to light damage and might lead to lethal consequences to the biochemical composition of vitreous fluid.

With the effects of blue, white, and yellow LEDs on the whole-mount retina, the significant structural alterations and damage leading to the disruption of the retina, could be due to disruption of blood-retinal barriers by increased production of ROS[47]. We found that the BLE and WLE exposed retinal tissues exhibited higher levels of CASPASE-3 immunostaining mostly in the INL, ONL and GCL of the retina (Fig. 5:) and this is consistent with previous findings [6, 30]. Furthermore, few studies found that post light exposure with higher intensity of blue and white LEDs inhibited the increased proliferation of retinal cells with apoptosis compared with yellow LED [6]. Unfortunately, studies have not been conducted for chronic light exposure (Beyond 28 days). The relationship between long-term light exposure and retinal damage was unidentified. Few studies have indicated that lipofuscin could be a possible mediator of the risk associated with long-term exposure to blue light-induced retinal damage $[48,49]$. When lipofuscin absorbs blue light, it produces ROS that eventually leads to oxidative stress and retinal damage [27]. This mechanism (lipofuscin) is directly related to the type of light and its spectral composition [50]. Thomas et al reported that chronic blue light exposure could accelerate photoreceptor degeneration in an animal model in a retinal degeneration study [51]. The lipofuscin levels in retinal pigmented epithelium may alter with age, with levels being low in young and high in old animals [52]. This could also be one of the confounding factors to accelerate the retinal damage along with chronic exposure to the blue and white component of the light spectrum. The presence of a self-protective mechanism diminishes with age [53]. Light exposure leads to oxidative stress eventually resulting in the visual system no longer being able to protect the retinal architectures against chronic light, thus triggering irreversible vagaries in the visual system. Overall, the attained results can suggest the following sequences of events the ROS produced by the high-energy light from a blue and white LED[54] and the altered vitreous metabolites by inducing protein and AA modification affecting the hypotaurine pathway. This observation suggests that photo-chemical metabolism is compromised in light exposure rats potentially disrupting taurine and hypotaurine metabolism that might impact neural signals in the retina Fig. 5 . This study has some limitations as we used only (CASPASE-3) immunohistological markers, which make little difference to the blue and white light delivered to the animals used in this study. A more detailed study, with more time point sampling and specific cell death assay (e.g. western blot), further reveal understated anatomical changes to use OCT (real-time imaging) in chronic light exposure. The results shown here were obtained in rats with cumulative and continuous LED light exposure, their eyes are different from humans because they are nocturnal animals and do not have a macula this cannot be directly applicable to humans.

\section{Conclusion}

Our findings state that chronic exposure to blue and white LED light damages the retina and alters the vitreous metabolites. The prolonged acute cumulative blue and white LED light exposure causes indemnities to the structural integrity of the vitreous and retinae. The self-protective or reworking system in the light-exposed visual system is dazed and deteriorate the ability to compensate for the self-protective mechanism.

\section{Declarations}

Funding: This research was funded by the Science\& Engineering Research Board (SERB) (a statutory body of the Department of Science \& Technology, Government of India), grant number EMR/2017/004341. 
Declaration of interests: The authors declare that they have no known competing financial interests or personal relationships that could have appeared to influence the work reported in this paper.

Author Contributions: All authors contributed to the study conception and design. Material preparation, data collection and analysis were performed by [Nagarajan T], [Manjunath B Joshi], [Sudarshan S], [Shonraj BG Rao], [Manna Valiathan] and [Shailaja S]. The first draft of the manuscript was written by [Nagarajan.T] and all authors commented on previous versions of the manuscript. All authors read and approved the final manuscript

Data Availability Statement: The data presented in this study are available on request from the corresponding author.

Ethical Statement: All experimental procedures in the present study were approved by the institutional animal ethics committee (IAEC) at Kasturba Medical College, Manipal Academy of Higher Education (IAEC/KMC/02/2017, dated 21 January 2017), India. Animal handling and investigational procedures were carried out as per CPCSEA (No:94/PO/Re Bi/5/99/ CPCSEA) and ARRIVE guidelines.

Informed Consent Statement: Not applicable.

\section{References}

1. Boettner EA (1962) Transmission of the Ocular Media. Investig Ophthalmol Vis Sci 1:776-783

2. Bailes HJ, Lucas RJ (2013) Human melanopsin forms a pigment maximally sensitive to blue light ( $\lambda \max \approx 479 \mathrm{~nm})$ supporting activation of $\mathrm{Gq} / 11$ and Gi/o signalling cascades. Proc R Soc B Biol Sci 280:. https://doi.org/10.1098/rspb.2012.2987

3. Al-Karawi D, Jubair L (2016) Bright light therapy for nonseasonal depression: Meta-analysis of clinical trials. J Affect Disord 198:64-71. https://doi.org/10.1016/j.jad.2016.03.016

4. Turner PL, Van Someren EJW, Mainster MA The role of environmental light in sleep and health: Effects of ocular aging and\&nbsp;cataract surgery. https://doi.org/10.1016/j.smrv.2009.11.002

5. Setlow RB, Grist E, Thompson K, Woodhead AD (1993) Wavelengths effective in induction of malignant melanoma. Proc Natl Acad Sci U S A 90:6666-6670. https://doi.org/10.1073/pnas.90.14.6666

6. Shang YM, Wang GS, Sliney DH, et al (2017) Light-emitting-diode induced retinal damage and its wavelength dependency in vivo. Int J Ophthalmol 10:191-202. https://doi.org/10.18240/ijo.2017.02.03

7. Sparrow JR, Miller AS, Zhou J (2004) Blue light-absorbing intraocular lens and retinal pigment epithelium protection in vitro. J Cataract Refract Surg 30:873-878. https://doi.org/10.1016/j.jcrs.2004.01.031

8. Ecker JL, Dumitrescu ON, Wong KY, et al (2011) cellular diversity and role in pattern vision. 67:49-60. https://doi.org/10.1016/j.neuron.2010.05.023.Melanopsin-expressing

9. Grimm C, Wenzel A, Williams TP, et al (2018) Rhodopsin-Mediated Blue-Light Damage to the Rat Retina: Effect of Photoreversal of Bleaching. 42:

10. Roehlecke $C$, Schumann U, Ader M, et al Influence of blue light on photoreceptors in a live retinal explant system

11. Wahnschaffe A, Haedel S, Rodenbeck A, et al (2013) Out of the Lab and into the Bathroom: Evening Short-Term Exposure to Conventional Light Suppresses Melatonin and Increases Alertness Perception. Int J Mol Sci 14:2573-2589. https://doi.org/10.3390/ijms14022573

12. Cheung IN, Zee PC, Shalman D, et al (2016) Morning and Evening Blue-Enriched Light Exposure Alters Metabolic Function in Normal Weight Adults. PLoS One 11:1-18. https://doi.org/10.1371/journal.pone.0155601

13. Ham WT, Mueller HA, Sliney DH (1976) Retinal sensitivity to damage from short wavelength light. Nature 260:153-155. https://doi.org/10.1038/260153a0

14. de la Barca JMC, Huang NT, Jiao H, et al (2017) Retinal metabolic events in preconditioning light stress as revealed by widespectrum targeted metabolomics. Metabolomics 13:. https://doi.org/10.1007/s11306-016-1156-9

15. Youssef PN, Sheibani N, Albert DM (2011) Retinal light toxicity. Eye 25:1-14. https://doi.org/10.1038/eye.2010.149 
16. Mainster MA, Ham WT, Delori FC (1983) Potential Retinal Hazards: Instrument and Environmental Light Sources. Ophthalmology 90:927-932. https://doi.org/10.1016/S0161-6420(83)80019-0

17. Natoli R, Jiao H, Barnett NL, et al (2016) A model of progressive photo-oxidative degeneration and inflammation in the pigmented C57BL/6J mouse retina. Exp Eye Res 147:114-127. https://doi.org/10.1016/j.exer.2016.04.015

18. Jaadane I, Villalpando Rodriguez G, Boulenguez P, et al (2020) Retinal phototoxicity and the evaluation of the blue light hazard of a new solid-state lighting technology. Sci Reports 2020101 10:1-13. https://doi.org/10.1038/s41598-020-63442-5

19. Chamorro E, Bonnin-Arias C, Pérez-Carrasco MJ, et al (2013) Effects of light-emitting diode radiations on human retinal pigment epithelial cells in vitro. Photochem Photobiol 89:468-473. https://doi.org/10.1111/j.1751-1097.2012.01237.x

20. Roehlecke C, Schumann U, Ader M, et al (2011) Influence of blue light on photoreceptors in a live retinal explant system. 876884

21. Hockberger PE, Skimina TA, Centonze VE, et al (1999) Activation of flavin-containing oxidases underlies light-induced production of $\mathrm{H} 2 \mathrm{O} 2$ in mammalian cells. Proc Natl Acad Sci U S A 96:6255-6260. https://doi.org/10.1073/pnas.96.11.6255

22. Contín MA, Arietti MM, Benedetto MM, et al (2013) Photoreceptor damage induced by low-intensity light: model of retinal degeneration in mammals. Mol Vis 19:1614-25

23. Kuse $\mathrm{Y}$, Ogawa $\mathrm{K}$, Tsuruma $\mathrm{K}$, et al Damage of photoreceptor-derived cells in culture induced by light emitting diode-derived blue light. https://doi.org/10.1038/srep05223

24. Smick K, Boulton ME, Grayson Professor of Ophthalmology M, et al (2013) Blue Light Hazard 2. 1-12

25. Boulton M, Rózanowska M, Rózanowski B (2001) Retinal photodamage. J Photochem Photobiol B Biol 64:144-161. https://doi.org/10.1016/S1011-1344(01)00227-5

26. Godley BF, Shamsi FA, Liang FQ, et al (2005) Blue light induces mitochondrial DNA damage and free radical production in epithelial cells. J Biol Chem 280:21061-21066. https://doi.org/10.1074/jbc.M502194200

27. Tosini G, Ferguson I, Tsubota K (2016) Effects of blue light on the circadian system and eye physiology. Mol Vis 22:61-72

28. LIGHTING PASSPORT |. https://lightingpassport.alliedscientificpro.com/? gclid=CjOKCQiAgebwBRDnARIsAE3eZjShUWLYTr3_M8CweK4kvwfoUNPssqguCbnHZuRpm3Pxbbke3qaJgVoaAgB9EALw_wcB. Accessed 11 Jan 2020

29. Behar-Cohen F, Martinsons C, Viénot F, et al (2011) Light-emitting diodes (LED) for domestic lighting: Any risks for the eye? Prog Retin Eye Res 30:239-257. https://doi.org/10.1016/j.preteyeres.2011.04.002

30. Liu X, Zhou Q, Lin H, et al (2019) The protective effects of blue light-blocking films with different shielding rates: A rat model study. TransI Vis Sci Technol 8:19-19. https://doi.org/10.1167/tvst.8.3.19

31. Thoreson WB, Witkovsky P (1999) Glutamate receptors and circuits in the vertebrate retina. Prog. Retin. Eye Res. 18:765-810

32. Izumi Y, Shimamoto K, Benz AM, et al (2002) Glutamate transporters and retinal excitotoxicity. Glia 39:58-68. https://doi.org/10.1002/glia.10082

33. Ishikawa M (2013) Abnormalities in Glutamate Metabolism and Excitotoxicity in the Retinal Diseases. Scientifica (Cairo) 2013:1-13. https://doi.org/10.1155/2013/528940

34. Sucher,- NJ, Lipton SA, Dreyer\$ EB (1997) Molecular Basis of Glutamate Toxicity Ganglion Cells in Retinal

35. Choi DW, Maulucci-Gedde M, Kriegstein AR (1987) Glutamate neurotoxicity in cortical cell culture. J Neurosci 7:357-368. https://doi.org/10.1523/jneurosci.07-02-00357.1987

36. Vorwerk CK, Lipton SA, Zurakowski D, et al Chronic Low-Dose Glutamate Is Toxic to Retinal Ganglion Cells Toxicity Blocked by Memantine

37. Laíns I, Gantner M, Murinello S, et al (2018) Metabolomics in the study of retinal health and disease. https://doi.org/10.1016/j.preteyeres.2018.11.002

38. Faktorovich EG, Steinberg RH, Yasumura D, et al (1990) Photoreceptor degeneration in inherited retinal dystrophy delayed by basic fibroblast growth factor. Nature 347:83-86. https://doi.org/10.1038/347083a0

39. Wen R, Cheng T, Song Y, et al (1998) Continuous exposure to bright light upregulates bFGF and CNTF expression in the rat retina. Curr Eye Res 17:494-500. https://doi.org/10.1076/ceyr.17.5.494.5186 
40. Song W, Zhang Y, Liu F, et al (2018) Taking advantage of multi-regions-based diagonal texture structure descriptor for image retrieval. Expert Syst Appl 96:347-357. https://doi.org/10.1016/j.eswa.2017.12.006

41. Organisciak DT, Darrow RM, Barsalou L, et al (2003) Susceptibility to retinal light damage in transgenic rats with rhodopsin mutations. Investig Ophthalmol Vis Sci 44:486-492. https://doi.org/10.1167/iovs.02-0708

42. Paul KN, Saafir TB, Tosini G The role of retinal photoreceptors in the regulation of circadian rhythms. https://doi.org/10.1007/s11154-009-9120-x

43. Barbe MF, Tytell M, Gower DJ, Welch WJ (1988) Hyperthermia protects against light damage in the rat retina. Science (80-) 241:1817-1820. https://doi.org/10.1126/science.3175623

44. Valderrama CM, Li R, Liu JHK (2008) Direct effect of light on 24-h variation of aqueous humor protein concentration in Sprague-Dawley rats. Exp Eye Res 87:487-491. https://doi.org/10.1016/j.exer.2008.08.015

45. Stastna M, Behrens A, Noguera G, et al (2007) Proteomics of the aqueous humor in healthy New Zealand rabbits. Proteomics 7:4358-4375. https://doi.org/10.1002/PMIC.200700300

46. Lavail MM, Unokit K, Yasumura D, et al (1992) Multiple growth factors, cytokines, and neurotrophins rescue photoreceptors from the damaging effects of constant light (light damage/retinal degeneration/photoreceptor rescue/neurotrophic factor/basic fibroblast growth factor). Neurobiology 89:11249-11253

47. Jaadane I, Villalpando Rodriguez GE, Boulenguez P, et al (2017) Effects of white light-emitting diode (LED) exposure on retinal pigment epithelium in vivo. J Cell Mol Med 21:3453-3466. https://doi.org/10.1111/jcmm.13255

48. Sparrow JR, Boulton M (2005) RPE lipofuscin and its role in retinal pathobiology. Exp. Eye Res. 80:595-606

49. Wolf G (2003) Lipofuscin and macular degeneration. Nutr. Rev. 61:342-346

50. Pawlak A, Różanowska M, Zareba M, et al (2002) Action spectra for the photoconsumption of oxygen by human ocular lipofuscin and lipofuscin extracts. Arch Biochem Biophys 403:59-62. https://doi.org/10.1016/s0003-9861(02)00260-6

51. Thomas BB, Seiler MJ, Aramant RB, et al (2007) Visual Functional Effects of Constant Blue Light in a Retinal Degenerate Rat Model. Photochem Photobiol 83:759-765. https://doi.org/10.1562/2006-09-19-ra-1044

52. Delori FC, Goger DG, Dorey CK (2001) Age-related accumulation and spatial distribution of lipofuscin in RPE of normal subjects. Investig Ophthalmol Vis Sci 42:1855-1866

53. Taylor HR, West S, Muñoz B, et al (1992) The Long-term Effects of Visible Light on the Eye. Arch Ophthalmol 110:99-104. https://doi.org/10.1001/archopht.1992.01080130101035

54. Narimatsu T, Negishi K, Miyake S, et al (2015) Blue light-induced inflammatory marker expression in the retinal pigment epithelium-choroid of mice and the protective effect of a yellow intraocular lens material invivo. Exp Eye Res 132:48-51. https://doi.org/10.1016/j.exer.2015.01.003

\section{Tables}

Table 1: The different wavelength of LED light exposure of individual retinal layer thicknesses stained with hematoxylin and eosin were measured using Image J software (RPE to RGC) layer thickness were measured at quarterly points for each retinal crosssection and averaged. Averaged retinal thickness was converted to the percentage of the thicknesses from keeping standard against controls. Also, the layers are examined with different parameters (Atrophy, vacuolation, pyknosis and morphological alteration) by histopathologist. 


\begin{tabular}{|c|c|c|c|c|}
\hline Layer & Damage & $\begin{array}{l}\text { BLE } \\
\mathrm{n}=12\end{array}$ & $\begin{array}{l}\text { WLE } \\
\mathrm{n}=12\end{array}$ & $\begin{array}{l}\text { YLE } \\
\mathrm{n}=12\end{array}$ \\
\hline \multirow[t]{4}{*}{ Ganglion Cell Layer } & Atrophy & $2 \%$ & $8 \%$ & $2 \%$ \\
\hline & Vacuolation & $4 \%$ & $6 \%$ & $4 \%$ \\
\hline & Proliferation & $7 \%$ & $1 \%$ & 0 \\
\hline & Focal enlargement & $8 \%$ & $10 \%$ & $6 \%$ \\
\hline Inner Plexiform Layer & Atrophy & $7 \%$ & 0 & 0 \\
\hline \multirow[t]{3}{*}{ Inner Nuclear Layer } & Focal enlargement & $4 \%$ & 0 & $4 \%$ \\
\hline & Atrophy & $2 \%$ & 0 & $2 \%$ \\
\hline & Decreased thickness & $2 \%$ & 6 & 0 \\
\hline \multirow[t]{4}{*}{ Outer Nuclear Layer } & Atrophy & 0 & 0 & $4 \%$ \\
\hline & Focal enlargement & 0 & 0 & $3 \%$ \\
\hline & Attenuated & 0 & 0 & $1 \%$ \\
\hline & Decreased thickness & $12 \%$ & $12 \%$ & $10 \%$ \\
\hline \multirow[t]{2}{*}{ Inner segment - Outer Segment } & Atrophy & $8 \% \%$ & $6 \% \%$ & $5 \%$ \\
\hline & Decreased thickness & 8 & $7 \%$ & $6 \%$ \\
\hline
\end{tabular}

Table 2: Distinguish altered vitreous metabolites in controls, BL, WL and YL Metabolite intensities of most abundant and significantly altered amino acids, small molecules and lipids detected in vitreous of control $(n=6), B L(n=6), W L(n=6)$ and $Y L(n=6)$. Statistically significant changes in metabolite intensity between the control and lens protection groups are represented by indicating $(* \star * p<0.001, * \star p<0.01, * p<0.05)$. 


\begin{tabular}{|c|c|c|c|c|c|c|c|c|c|}
\hline \multirow[t]{2}{*}{ S.no } & \multirow[t]{2}{*}{ Mass } & \multirow[b]{2}{*}{ Metabolites } & \multirow{2}{*}{$\begin{array}{l}\text { P-Value - } \\
\text { ANOVA }\end{array}$} & \multicolumn{6}{|c|}{ Tukey HSD post-hoc analysis } \\
\hline & & & & $\begin{array}{l}\text { NC- } \\
\text { BLE }\end{array}$ & $\begin{array}{l}\text { BLE- } \\
\text { WLE }\end{array}$ & $\begin{array}{l}\text { BLE- } \\
\text { YLE }\end{array}$ & $\begin{array}{l}\text { NC- } \\
\text { WLE }\end{array}$ & $\begin{array}{l}\text { NC- } \\
\text { YLE }\end{array}$ & $\begin{array}{l}\text { WLE- } \\
\text { YLE }\end{array}$ \\
\hline 1 & 89.0932 & D-Alanine & 0.013 & 0.02 & 1 & 0.753 & 0.021 & 0.146 & 0.773 \\
\hline 2 & 105.0926 & D-Serin & 0.009 & 0.091 & 0.016 & 0.014 & 0.84 & 0.811 & 1 \\
\hline 3 & 119.1192 & L-Threonine & $<0.001$ & 0.002 & 0.503 & 0.629 & $<0.001$ & $<0.001$ & 0.997 \\
\hline 4 & 125.147 & Taurine & 0.006 & 0.861 & 0.006 & 0.16 & 0.032 & 0.509 & 0.401 \\
\hline 5 & 128.172 & lysine & 0.001 & 0.014 & 0.733 & 0.89 & 0.001 & 0.003 & 0.989 \\
\hline 6 & 132.1577 & L-Asparagine & 0.011 & 0.051 & 0.987 & 0.943 & 0.025 & 0.016 & 0.996 \\
\hline 7 & 133.1027 & D-aspartic acid & 0.012 & 0.122 & 0.975 & 0.021 & 0.25 & 0.818 & 0.05 \\
\hline 8 & 147.13 & L-glutamic acid & $<0.001$ & $<0.001$ & 0.509 & 0.857 & 0.009 & 0.002 & 0.926 \\
\hline 9 & 153.1784 & Dopamine & 0.009 & 0.091 & 0.016 & 0.014 & 0.84 & 0.811 & 1 \\
\hline 10 & 175.188 & citrulline & $<0.001$ & $<0.001$ & 0.734 & 0.995 & 0.002 & $<0.001$ & 0.596 \\
\hline 11 & 219.237 & Pantothenate & 0.017 & 0.096 & 0.92 & 0.94 & 0.026 & 0.03 & 1 \\
\hline 12 & 228.245 & Hydroxyprolyl-Proline & 0.004 & 0.053 & 0.619 & 0.975 & 0.004 & 0.022 & 0.851 \\
\hline 13 & 231.249 & L-Asparaginyl-L-valine & 0.004 & 0.979 & 0.994 & 0.02 & 0.999 & 0.008 & 0.012 \\
\hline 14 & 231.296 & $a-(Y-A m i n o b u t y r y l)-l y s i n e$ & 0.02 & 0.338 & 0.414 & 0.778 & 0.017 & 0.064 & 0.923 \\
\hline 15 & 284.272 & L-Glutamyl-L-histidine & 0.038 & 0.22 & 0.849 & 0.46 & 0.049 & 0.956 & 0.131 \\
\hline 16 & 307.32 & Glutathione & 0.012 & 1 & 0.019 & 0.743 & 0.02 & 0.757 & 0.144 \\
\hline 17 & 317.3398 & $\begin{array}{l}\text { L-Hydroxyprolyl-L- } \\
\text { tryptophan }\end{array}$ & $<0.001$ & 0.002 & 0.965 & 0.999 & 0.001 & 0.001 & 0.989 \\
\hline 18 & 364.5619 & 2-Arachidonylglycerol & 0.041 & 0.067 & 0.999 & 0.87 & 0.05 & 0.263 & 0.802 \\
\hline 19 & 420.0956 & $\begin{array}{l}\text { inositol 1,4,5- } \\
\text { trisphosphate }\end{array}$ & 0.029 & 0.176 & 0.826 & 0.935 & 0.033 & 0.059 & 0.993 \\
\hline 20 & 579.7895 & Lysophosphatidylcholine & 0.011 & 0.819 & 0.01 & 0.139 & 0.066 & 0.514 & 0.598 \\
\hline
\end{tabular}

Figures 


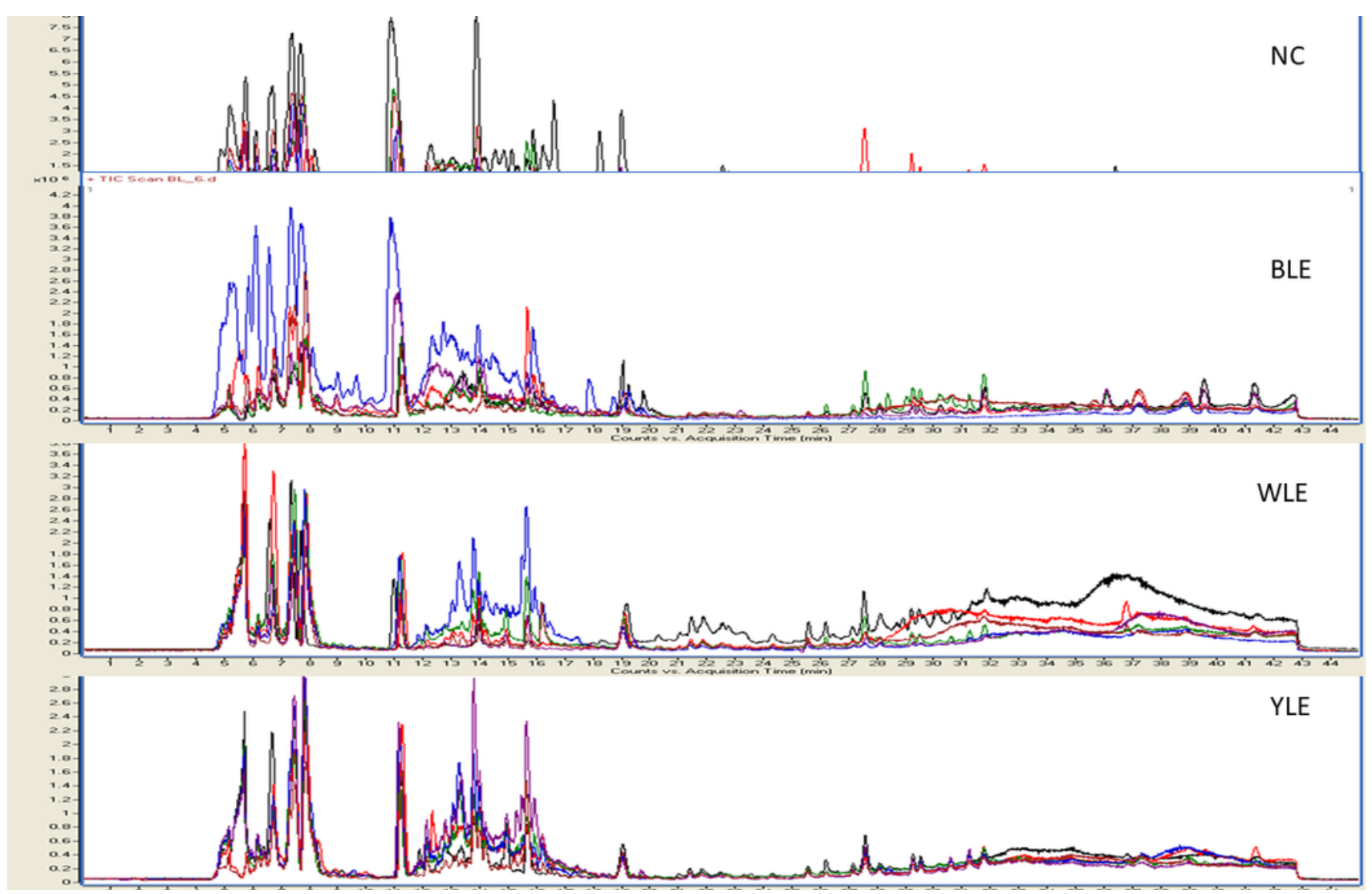

Figure 1

Represents the spectra features of metabolite peaks were obtained in NC, BLE, WLE and YLE groups separately. The X-axis represents the count versus acquisition of metabolites time in minutes and $\mathrm{Y}$-axis represents the generated distinct peaks on the total ion chromatogram (TIC) traces $\left({ }^{\star} 10^{6}\right)$ 

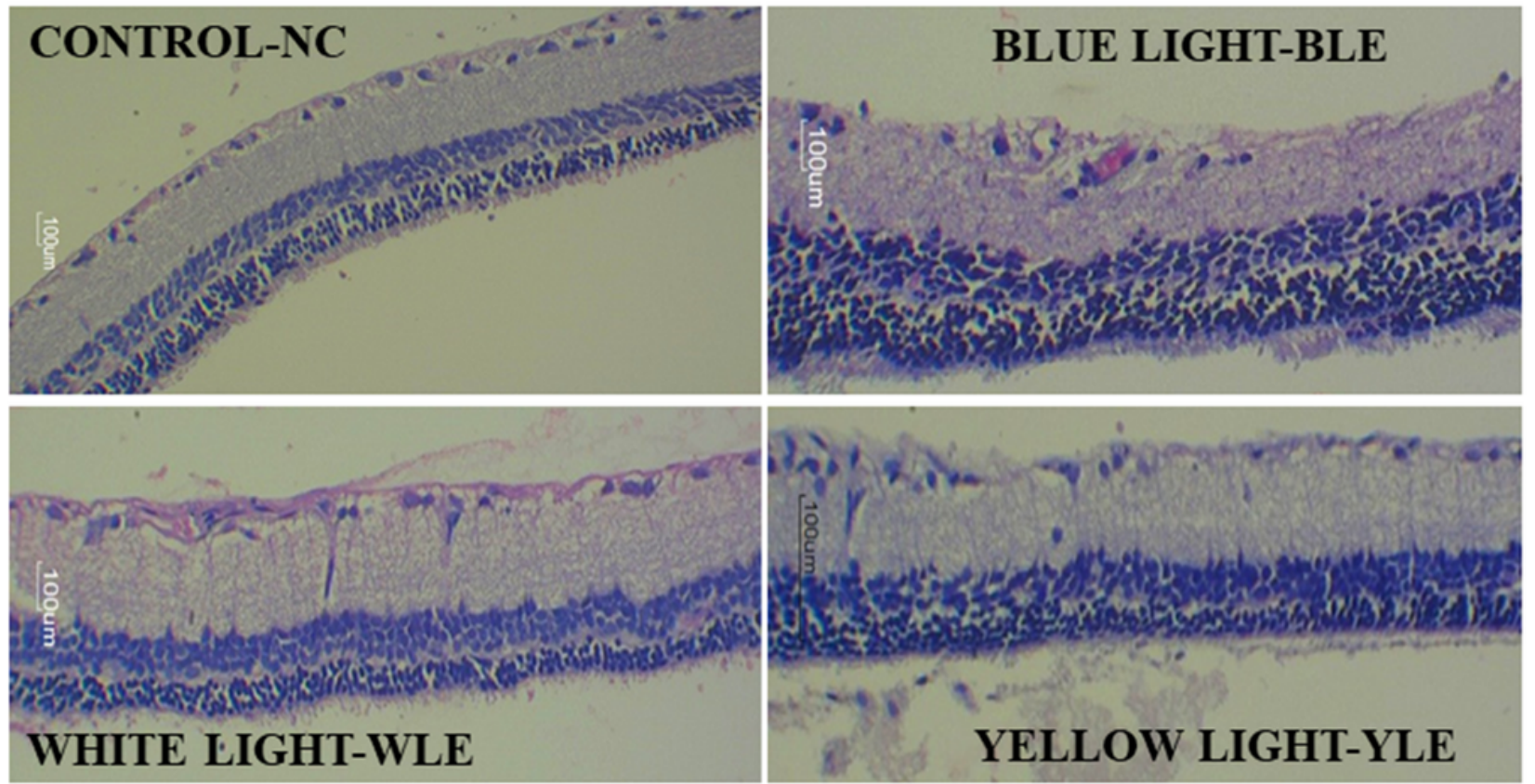

Figure 2

Retinal Histology of normal controls (NC), blue light exposure (BLE), white light exposure (WLE) and yellow light exposure (YLE) groups after 90 days of light exposure. Representative images of Hematoxylin and Eosin-stained retinal cross-sections from the Controls (NC.), blue light (BLE), white light (WLE) as well as yellow light (YLE) groups. Scale bar: $100 \mu \mathrm{m}$. Photoreceptor, Ganglion cell layer (GCL), inner nuclear layer (INL), inner plexiform layer (IPL), outer plexiform layer (OPL), and outer nuclear layer (ONL). 
Figure 3A:
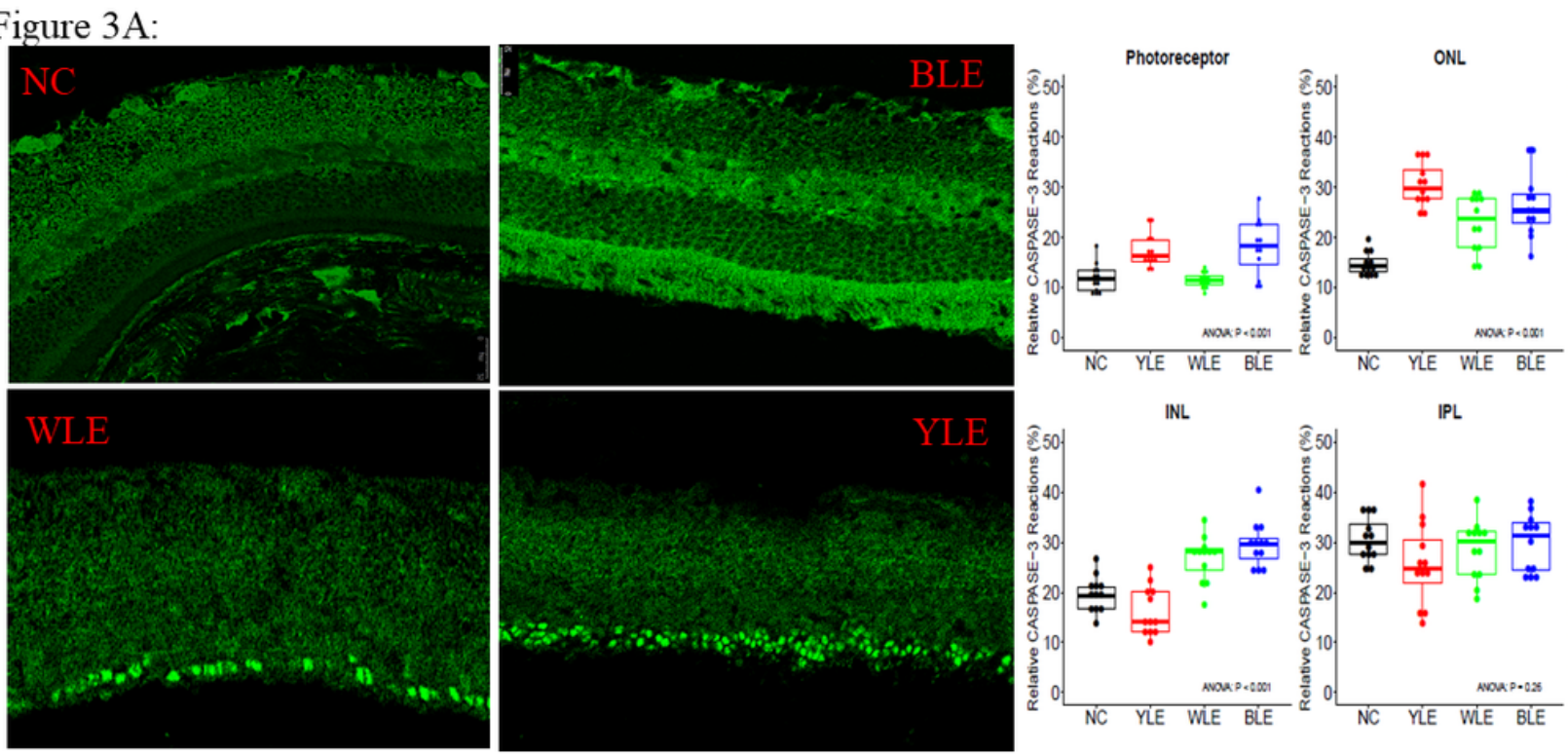

Figure $3 B$ :
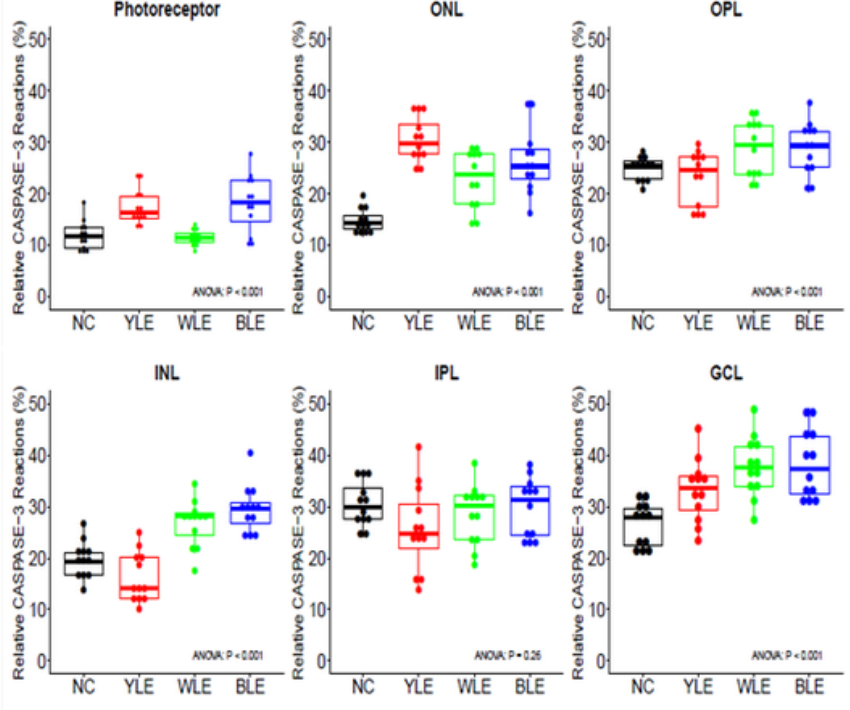

GCL

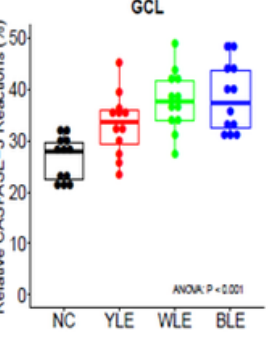

\section{Figure 3}

A: Different wavelengths of LED light-induced caspase-3 immunostaining in retinal layers. Representative images of retinal cryosections from normal control (NC top right), light exposure (BLE, top left) and WLE groups second row right and YLE second row left) immunolabeled with cleaved caspase-3 antibody (Immunofluorescence), IgG (whole molecule) F(ab)2 fragment-Cy3 antibody). At 90 days after light exposure, BLE and WLE eyes showed increased apoptosis. This was less evident of caspase-3 levels in NCs and YLE groups.

B: Effect of different LED light exposure on retinal caspase immunofluorescence. Bar plots showing immunofluorescence intensity relative to total retinal intensity (\%) for the ganglion cell layer. Error bars show the standard error of the mean. P-values in each panel indicates the significance of one-way ANOVA comparison between groups. * Indicates significant $(p<0.05)$ post-hoc difference between pairs. 


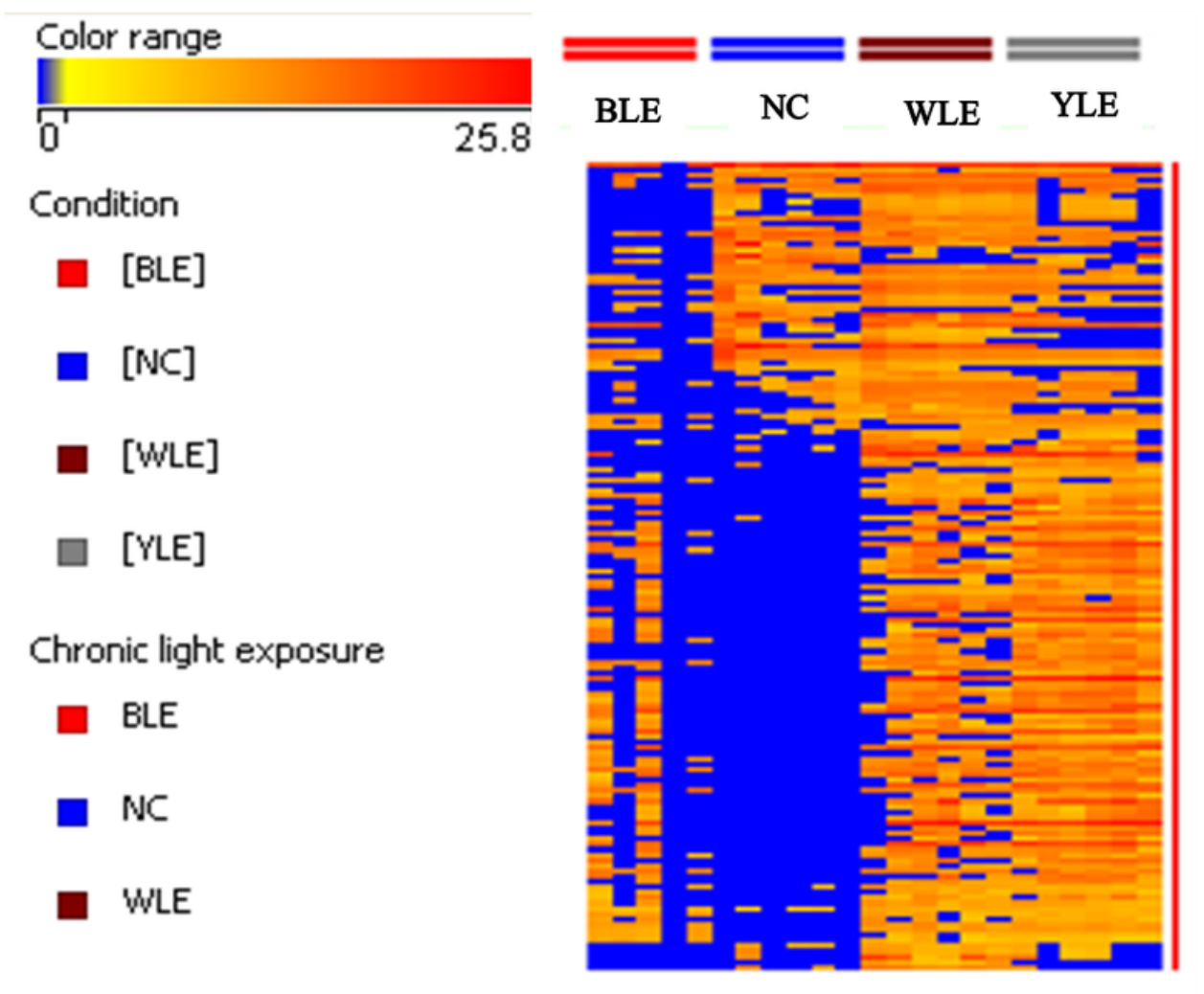

\section{Figure 4}

Hierarchical clustering analysis (HCA) by R version. a. HCA of all vitreous metabolites. b. HCA of significant Metabolites across the group. The colour gradient illustrates the fold change ( $F$ c3.1) of protein abundance between the four groups. A clear separation of the metabolite's alteration in the three exposure and control group conditions is shown and the alteration becomes more ostensible when significant metabolites have been considered. 

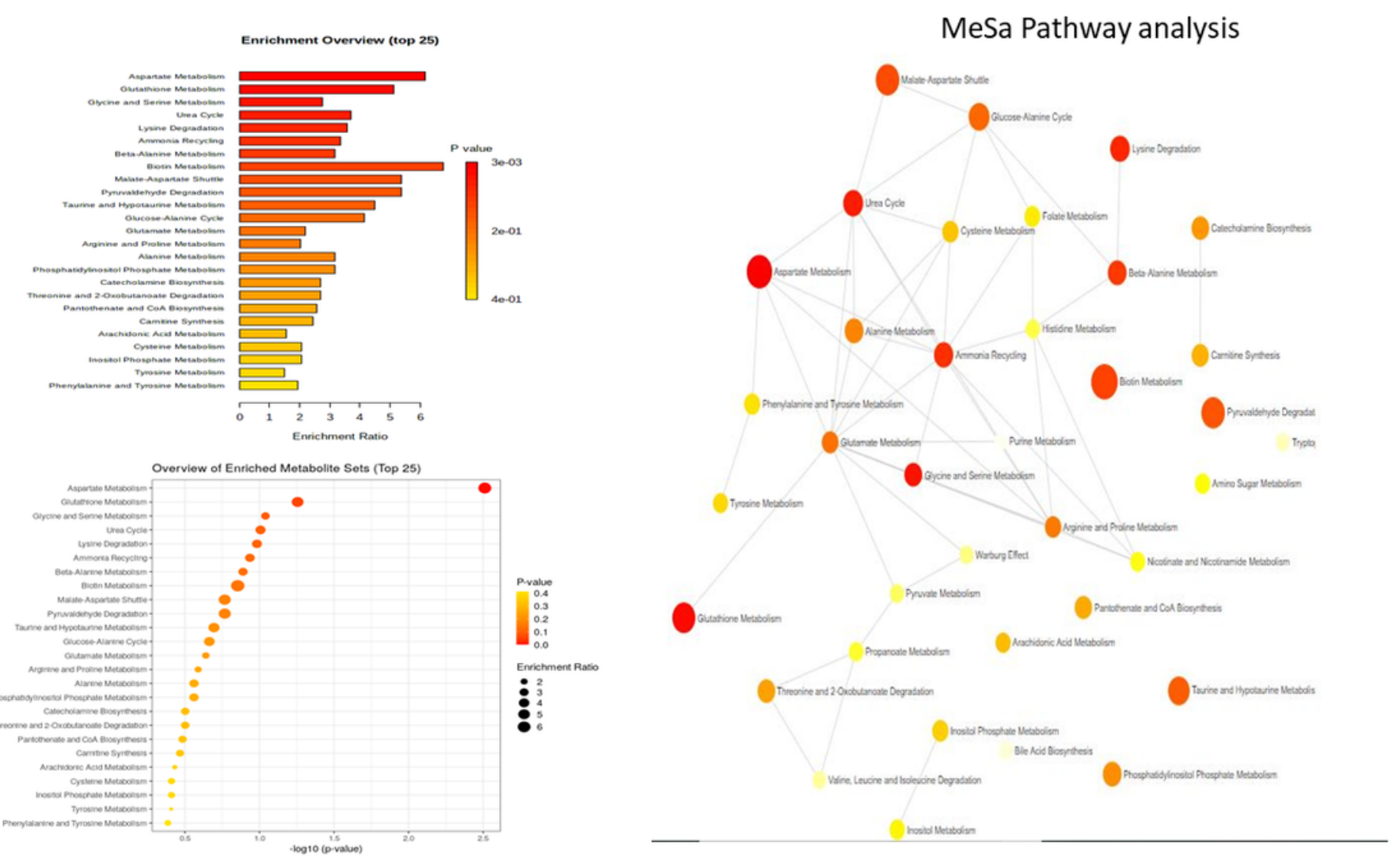

Figure 5

Metabolite set enrichment analysis: The Aspartate, Glycine, Serine, and glutathione metabolic pathways are the most significantly affected in the upper panel; 4 Upregulated FDR = 2.99E-01 and 4/20; FDR = 1.00E+00) and in the lower panel; 4 dysregulated features out of 20; False discovery rate $(F D R)=1.00 E+00$ ) 